\title{
Implementation of Automated Tube Current Modulation in PET/CT: Prospective Selection of a Noise Index and Retrospective Patient Analysis to Ensure Image Quality
}

\author{
James Jackson ${ }^{1}$, Tinsu Pan ${ }^{2}$, Elena Tonkopi ${ }^{2}$, Nancy Swanston ${ }^{1}$, Homer A. Macapinlac ${ }^{1}$, and Eric M. Rohren ${ }^{1}$ \\ ${ }^{1}$ Department of Nuclear Medicine, University of Texas M.D. Anderson Cancer Center, Houston, Texas; and ${ }^{2}$ Department of Imaging \\ Physics, University of Texas M.D. Anderson Cancer Center, Houston, Texas
}

\begin{abstract}
Automated tube current modulation (ATCM) has been shown to be a useful tool for reducing CT dose. However, its implementation can be complicated, because the correlation between noise index (NI) settings and noise production can change as parameters are manipulated. The goal was to create a methodology to prospectively select ATCM parameters and retrospectively ensure consistent image quality. Methods: An anthropomorphic phantom was scanned at various NIs to determine a baseline $\mathrm{NI}$ versus image noise. The noise was measured in SDs of the CT number reported in Hounsfield units. A physician then reviewed 45 studies performed with the same fixed-tube-current protocol to obtain a preferred noise level. The noise level was compared with our phantom baseline scans to find a suitable $\mathrm{NI}$ value. This value was implemented in clinical operation. Then, the next 50 patient examinations were retrospectively reviewed to ensure that image quality was maintained to our physician's cutoff noise levels. Radiation dose reductions through tube current reduction were measured for all CT slices of each patient study. Results: In the phantom study, tube current modulation was observed at an $\mathrm{NI}$ of 15 . The preferred noise level established in the physician's review correlated with an $\mathrm{NI}$ of 20 . In our postimplementation analysis, we found that our noise level was 10.75 SDs in Hounsfield units. CT dose reductions of up to $52 \%$ were seen. Conclusion: We were able to prospectively select an NI for ATCM CT by correlating phantom scans to a physician's preferred noise level while maintaining consistent image quality.
\end{abstract}

Key Words: automated tube current modulation; PET/CT; dose reduction

J Nucl Med Technol 2011; 39:83-90

DOI: 10.2967/jnmt.110.075283

\footnotetext{
Received Jul. 18, 2010; revision accepted Nov. 17, 2010.

For correspondence or reprints contact: James Jackson, Department of Nuclear Medicine, Unit 1264, University of Texas M.D. Anderson Cancer Center, 1515 Holcombe Blvd., Houston, TX 77030.

E-mail: James.jackson@mdanderson.org

COPYRIGHT @ 2011 by the Society of Nuclear Medicine, Inc.
}

$\mathbf{P}$ ET has for decades produced physiologic images that have changed disease management in cancer and cardiac patients $(1,2)$. In the past decade, the introduction of in-line CT for attenuation correction and anatomic localization has increased the sensitivity and specificity of PET in numerous oncologic applications (3). With this improved accuracy, the use of PET increased $10.4 \%$ annually in the United States from 2005 to 2008. Although this growth has slowed somewhat over the past couple of years to single digits (4), the use of PET is expected to continue to expand.

Although PET/CT produces more accurate and efficient examinations than does PET alone, CT acquisition introduces a second source of radiation exposure. Brix et al. analyzed 4 university hospitals' PET/CT protocols and found that each scan delivered approximately $25 \mathrm{mSv}$ to the patient, with CT and PET accounting for 18 and $7 \mathrm{mSv}$, respectively (5). However, CT use in PET/CT acquisition varies from one institution to another. In some institutions, CT images are used strictly for attenuation correction in the PET image and thus impart a very low radiation dose to the patient. In other institutions, CT images are acquired with diagnostic quality, with or without contrast material, thereby resulting in a large radiation dose to the patient.

Traditional CT techniques use a tube current (measured in milliamperes) that is fixed-that is, remains constant throughout the entire scan. One limitation of fixed-tubecurrent protocols is that image quality degrades in larger patients versus smaller patients. Furthermore, improvement in image quality appears to provide no particular benefit to diagnostic accuracy in the smaller patients, scanned with the same technique as larger patients. Weight- and agebased fixed-tube-current protocols have been tested and advocated, especially in pediatric populations (6), but the aforementioned issues may still persist because weight distribution is not always uniform among patients with similar weights or body mass indices (BMIs). A more recent approach to addressing these issues is the use of automated tube current modulation (ATCM). 
In ATCM, the tube current is adjusted during CT acquisition to deliver the most effective dose while achieving a predefined noise level. Over the past decade, ATCM has been shown to produce diagnostic-quality images while significantly lowering the radiation doses in diagnostic CT procedures. Livingstone et al. showed that ATCM provided a radiation dose saving over that of weight-based protocols while delivering diagnostic images (7). ATCM is reported to be capable of producing uniform noise in cardiac CT scans (8). Additional studies detail noise changes (measured in Hounsfield units [HU] as SDs of the CT number) as the CT technique is varied and as the ATCM settings are manipulated (9). Each manufacturer supports a version of ATCM and recommended program settings. For example, the Smart mA (GE Healthcare) program's settings are adjusted by changing a noise index (NI) value, which is based on SDs of the CT number in a water phantom using a standard reconstruction algorithm. The NIs are preset and are used to modulate the tube current to a set maximum to achieve a specified noise level. Kalra et al. detailed this and other ATCM methods (10).

There has been some confusion in the radiologic community over how to select an NI, as the correlation between NI value and image noise is not well understood. According to GE Healthcare, the NI is designed to correlate to the SD measurement in a water phantom with a standard reconstruction. However, the parallel achieved with the default setting for Smart mA does not adapt as parameters such as spin time or pitch are manipulated. These changes were demonstrated by Rampado et al. (9). Furthermore, if an imaging facility uses a different reconstruction algorithm (soft vs. standard), this will change the SDs obtained for a particular NI. Thus, unless an institution follows all the manufacturer's guidelines for CT scan parameters, the NI will not be equal to the noise (SDs) found in the scan. Further analysis is needed to determine the noise level that will be produced by a deviation from the manufacturer's recommended parameters so an NI can be prescribed to achieve a predetermined level of tolerable image noise.

Although numerous authors have examined the effects of current modulation on radiation dose savings and image quality in phantom and patient scans $(8-11)$, in a literature search none was found that prospectively identified an NI on the basis of physician preference and the image quality of phantom scans. For example, Kalra et al. found their settings for NI in chest studies by retrospectively analyzing images scanned with NIs of 10.0, 12.5, and 15.0 (11). They found that examinations done with NIs of 10.0 and 12.5 using a standard reconstruction algorithm were of acceptable quality and resulted in dose savings of $18 \%$ and $26 \%$, respectively, over examinations done using their fixed-tubecurrent protocol.

In this study, we assessed a methodology for prospectively selecting the NI for the Smart mA program, because our protocol differs from the manufacturer's recommended parameters in pitch (GE Healthcare recommends a pitch of
0.938; our pitch setting is 1.375 ). The feasibility of setting the NI on the basis of a physician's preferred noise level was also tested. Finally, after the implementation of our predictive NI settings for the ATCM protocol, a retrospective analysis was evaluated to ensure that target values were achieved and image quality was maintained.

\section{MATERIALS AND METHODS}

Baseline testing was performed using an anthropomorphic phantom to analyze the change in noise (measured in $\mathrm{SD}$ of $\mathrm{HU}$ ) relative to NI selection in the standard CT protocol. A physician assessed fixed-tube-current patient studies to find a personalized preferred noise level. From this assessment, determination of a threshold to correlate with an NI from the phantom scans was attained. This NI was then implemented in the clinic, and postimplementation analysis was conducted to determine the radiation dose reductions and noise levels for scans of the liver and thorax.

\section{Phantom Scans}

A phantom study was performed to establish a baseline NI relative to noise, measured in SD of $\mathrm{HU}$, to be used as a template to prescribe a value once the physician's preferred noise threshold was documented.

An ATOM anthropomorphic phantom (head, thorax, abdomen, and pelvis, without arms; CIRS) was scanned in a supine position on a 16-slice LightSpeed (Discovery STE PET/CT; GE Healthcare) scanner. The phantom was positioned at isocenter and marked at the superior aspect of the cranium. A posterior-anterior scout image was then obtained $(120 \mathrm{kV}, 10 \mathrm{~mA})$, and baseline helical CT of the entire phantom was performed $(120 \mathrm{kV}, 300 \mathrm{~mA}, 0.5-\mathrm{s}$ gantry rotation, pitch of $1.375: 1$, x-ray collimation of $16 \times$ $1.25 \mathrm{~mm}$ ). Then, without moving the phantom or reestablishing the landmarks, we obtained consecutive CT scans using the Smart mA program for $x$-, $y$-, and $z$-axis modulation for the following NIs: 10.0, 12.5, 15.0, 17.5, 20.0, 22.5, and 25.0.

The images were sent to an Advantage workstation (4.3; GE Healthcare) for analysis. Each examination set had images for 6 regions of interest (ROIs) with a minimal area of $200 \mathrm{~mm}^{2}$. Three ROIs were placed in the thorax, and an additional 3 in the liver. The mean noise levels of the liver ROIs were plotted against their respective NIs. The practice was duplicated for the ROIs in the chest.

\section{Retrospective Analysis of Clinical \\ Fixed-Tube-Current Images}

In an analysis to find the physician's preferred noise level, images from 45 patient examinations acquired using our standard low-dose, fixed-tube-current $(120 \mathrm{kV}, 300$ $\mathrm{mA}$ ) protocol were selected so that there were 5 patients in each of the following BMI ranges: less than 20.0, 20.0 $22.4,22.5-24.9,25.0-27.4,27.5-29.9,30.0-32.4,32.5-$ $34.9,35.0-37.4$, and 37.5 or more. Only patients who had been imaged with their arms up were included. For each patient, 3 ROIs in the thorax (1 in the aortic arch and 2 in 

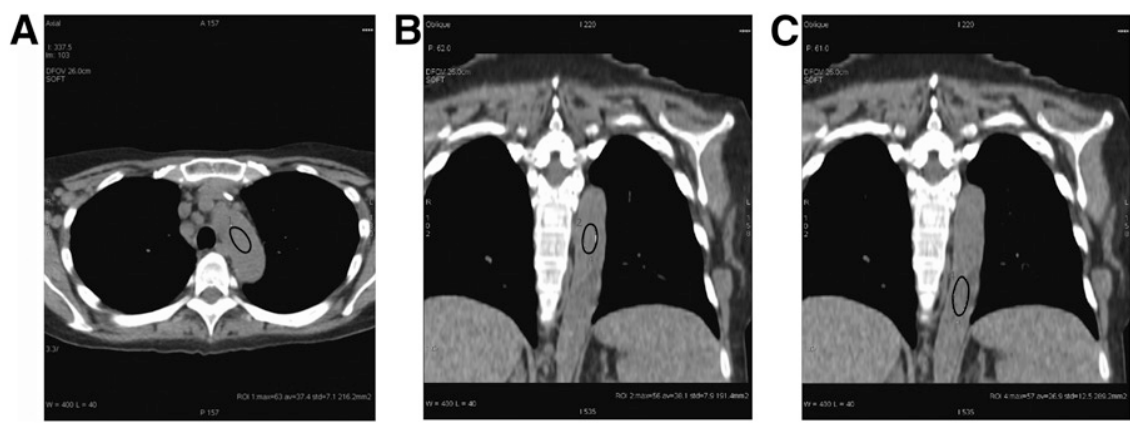

FIGURE 1. ROls in patient's thorax for SD measurement with minimal area of $200 \mathrm{~mm}^{2}$ : axial slice in aortic arch (A), descending aorta above liver in coronal view (B), and descending aorta at level of liver (C).

the descending aorta; Fig. 1) and 3 ROIs in the liver (dome, body of the liver where no lung tissue was visible, and in the most inferior aspect of the liver; Fig. 2) were analyzed for image noise relative to patient BMI.

The patient identification data on these images were removed; the images were randomly numbered and sent to an Advantage Workstation, where each was reviewed by a physician who is board-certified in both radiology and nuclear medicine. The physician was asked to judge each image according to noise level for an unenhanced CT image. The images were graded in 3 areas (thorax, liver, and overall) on a scale of 1-3. A score of 1 signified noise that would inhibit the physician's ability to locate anatomy for correlation with PET findings. A score of 2 signified an image that allowed for anatomic localization but was not considered to be of diagnostic quality. A score of 3 indicated diagnostic quality.

These scores were plotted against the noise levels of their respective scans. To compensate for the subjective nature of physician image quality grading, a linear regression model was used to calculate the line of best fit.

\section{Postimplementation Patient Image Analysis}

A postimplementation analysis was completed to ensure the system's ability to maintain the NI relative to noise. After the new protocol was implemented at the institution, images from 50 consecutive patients with a BMI of less than 30 who had been imaged with their arms up were analyzed mirroring the retrospective study to ensure that the predicted noise level was attained. The mean of the noise levels of the 3 thorax ROIs and the mean of the noise levels of the 3 liver ROIs were calculated and checked against the expected range of noise levels.

\section{Tube Current Analysis}

The radiation dose reduction resulting from tube current reduction was estimated for CT images of 370 patients (including the 50 patients in the postimplementation analysis) with an in-house program that extracted the tube current from each individual slice. The average current per slice was then calculated, and the resulting number was divided by 300 (the number of milliamperes of our fixed tube current) and subtracted from 1. This number was then multiplied by 100 to get the amount of dose reduction as a percentage of the dose from our fixed-tube-current protocol.

\section{RESULTS}

\section{Phantom Results}

No modulation was observed until an NI of 15 was implemented using the baseline parameters (Fig. 3). A change in modulation was determined by proprietary in-house software that allowed us to see if there were any changes in amperage during the CT acquisition. The program pulled the reported tube current in each slice from the examination. Any deviation below $300 \mathrm{~mA}$ was attributed to ATCM.

\section{Retrospective Analysis of Clinical \\ Fixed-Tube-Current Images}

There was a moderate correlation between patient BMI and noise $(r=0.82)$ in images of both the thorax ROIs and the liver ROIs (Fig. 4).
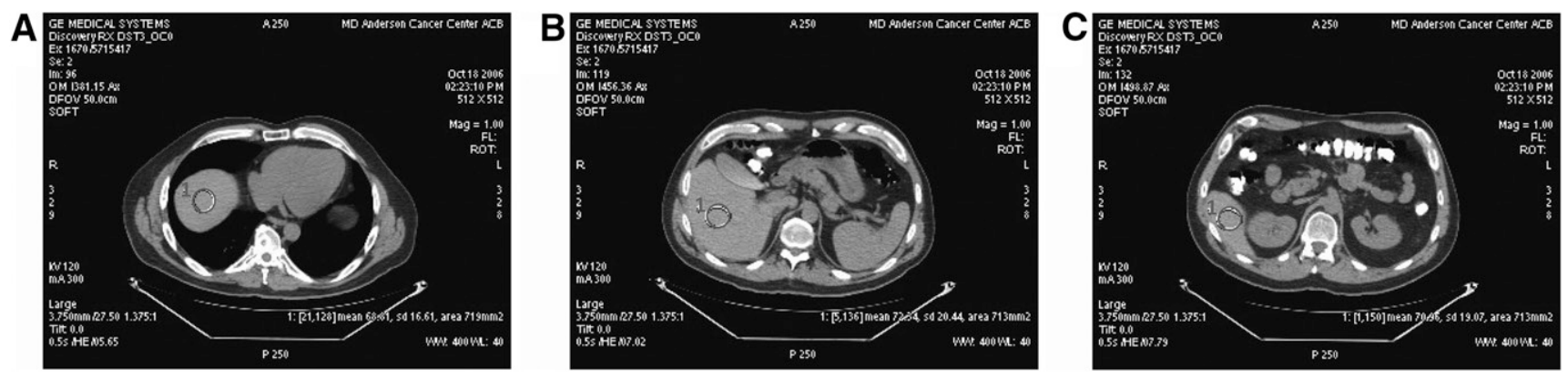

FIGURE 2. ROls in patient's liver for SD measurement with minimal area of $200 \mathrm{~mm}^{2}$ : dome of liver (A), middle level of liver, where no lung tissue can be seen $(B)$, and tail of liver $(C)$. 
FIGURE 3. Average noise (mean of SDs of CT numbers in $\mathrm{HU}$ ) in CT images at various $\mathrm{NI}$ settings for phantom acquisitions of 3 ROls in thorax and 3 in liver.

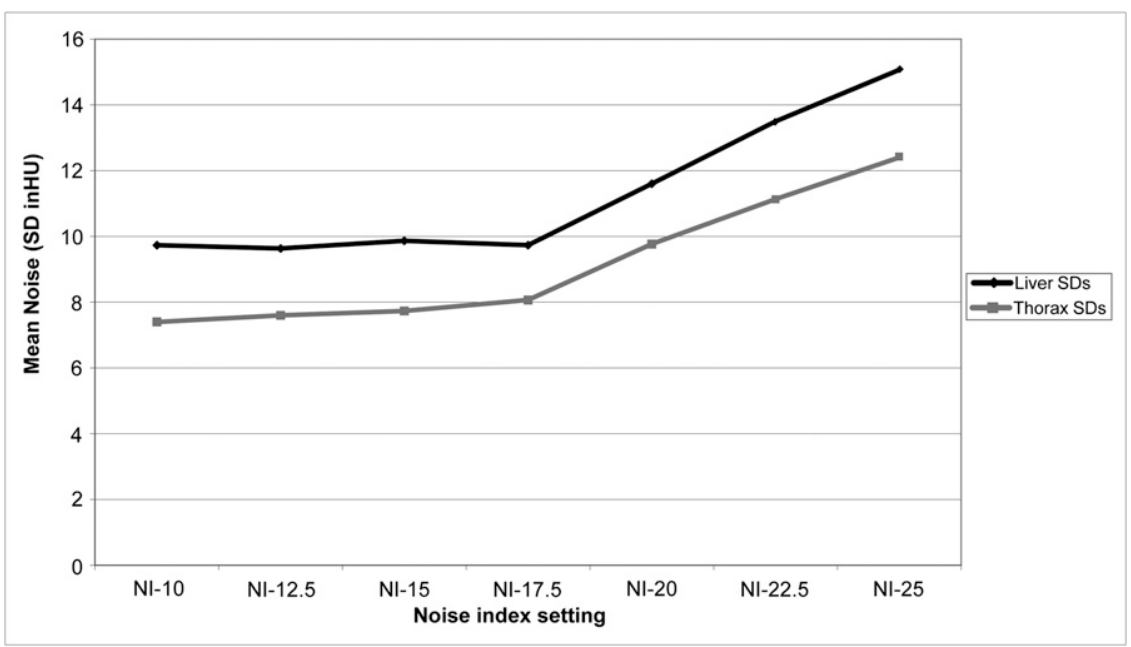

The endpoint for the physician's quality assessment of the patient images was to find the noise levels at which the best-fit line equaled a physician score of 2.75 . The best-fit line equaled 2.75 at $10-11$ SDs in HU for images of the thorax and 12-13 SDs in HU for images of the liver (Fig. 5); therefore, these were considered the cutoff noise levels for diagnostic-quality images.

The physician's cutoff noise level of 12-13 SDs in HU for scans of the liver corresponded to an NI of 22.5 in the phantom scans. The thorax was determined to be the region in which the physician was least tolerant of noise, with a cutoff noise level of 10-11 SDs in HU, which corresponded to an NI of 20 in the phantom scans. Therefore, because the cutoff noise levels for thorax scans were determined to be the limiting factor, an NI of 20 was implemented into standardized clinical practice (Fig. 6).

\section{Postimplementation Patient Image Analysis}

When the NI was set to 20, a trend for tube current modulation to occur in scans of patients with BMIs of 28 or less was detected. For these patients, there was a consistent mean noise level of about 10.75 HUs in images of the thorax, which fell within the target range of 10-11 HUs (Table 1). Also in patients with BMIs of 28 or less, a mean SD of 11.5 HUs was noted in images of the liver, which correlated to the noise level predicted by the phantom scans of the liver with an NI of 20. Some tube current modulation was detected in total body scans of patients with BMIs higher than 28, but this modulation was not observed in images of the thorax or abdomen. The tube current modulation here was due to a change in the head and neck region.

\section{Tube Current Reduction}

The reduction in tube current was in proportion to BMI (Fig. 7). The tube current reduction was as great as $52 \%$. No significant tube current reductions were found in any images of patients with BMIs of 33 or more, and no significant tube current reductions were found in images of the thorax or abdomen in patients with BMIs of 28 or more. The slight tube current reductions in images of patients with BMIs higher than 30 was attributable to dose reduc-
FIGURE 4. Average image noise (SD of CT number in $\mathrm{HU}$ ) for CT images of 3 ROls in thorax and 3 in liver for 45 patients analyzed retrospectively and grouped according to BMl. Avg = average.

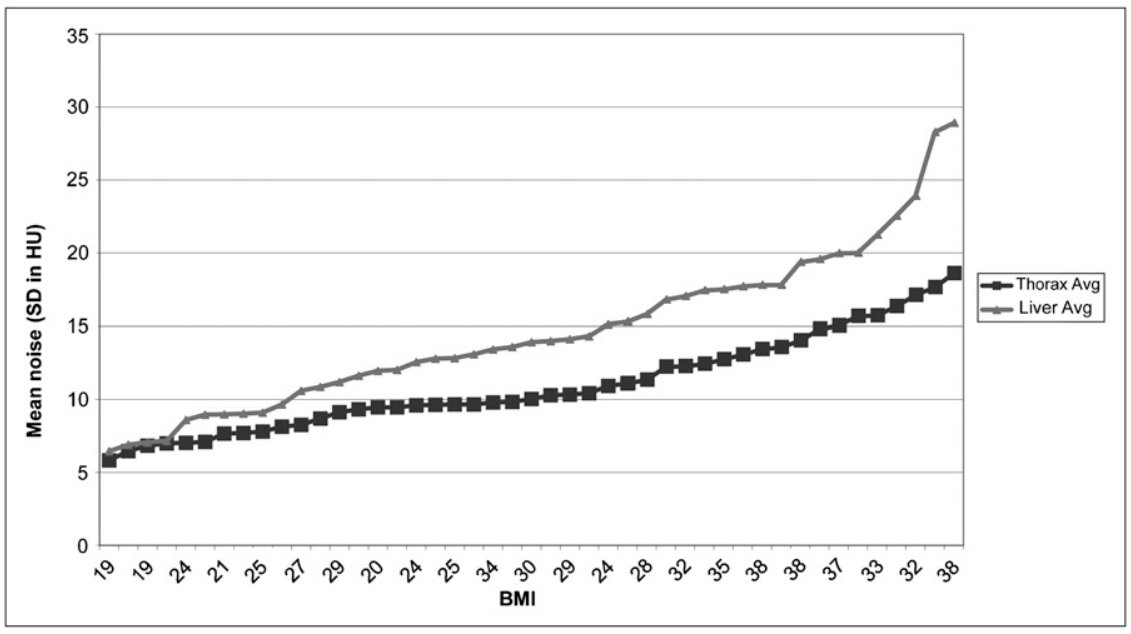



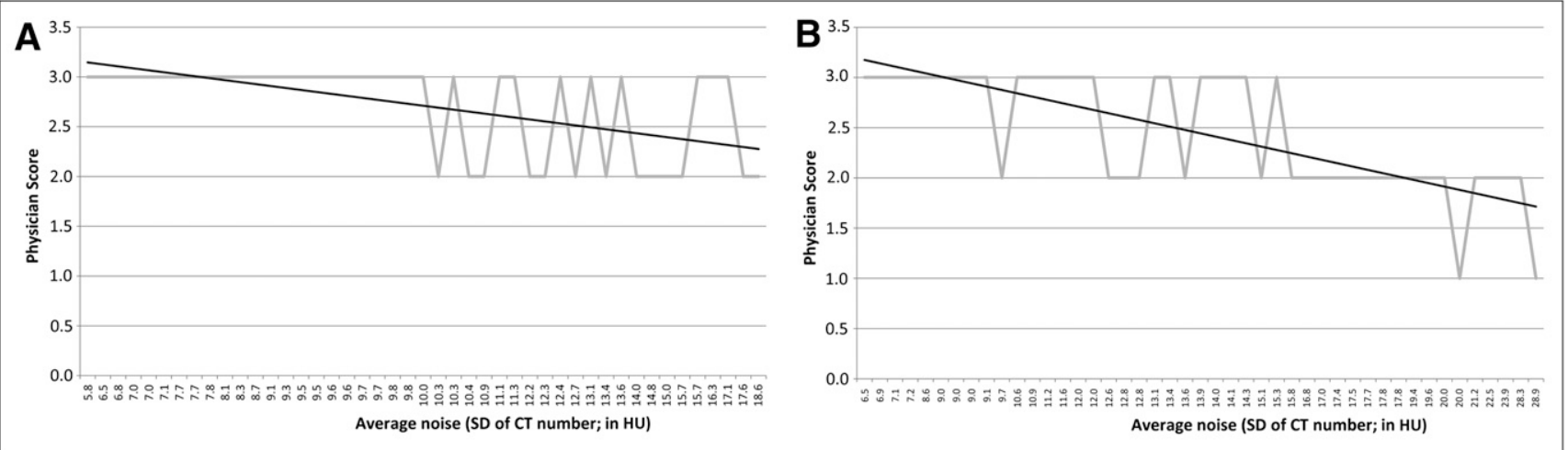

FIGURE 5. Physician-determined CT image quality (on scale of 1-3) according to noise level in scans of thorax (A) and scans of liver (B). Black lines indicate best-fit linear functions for data.

tions seen in the cranial region of patients who had undergone total-body scans.

\section{DISCUSSION}

The results show that it is possible to prospectively select an NI for ATCM CT by correlating phantom scans to a physician's preferred noise level. Consistency is key in this process. Various studies reported that changing the type of scout used (e.g., lateral vs. anteroposterior) or choosing a certain pitch over another can be detrimental to image quality $(9,12)$. But if these changes are factored in to the initial parameters and tested, the changes should not be detrimental. It is not enough to simply apply an NI and allow for various changes to technique (e.g., pitch or spin), because these will change the way the dose is delivered and thus the level of noise presented in an image. Imaging facilities electing to use and implement an ATCM protocol should focus on how much noise is reasonable and whether adopting another site's CT parameters, including spin, pitch, and kilovoltage, is acceptable for the patient population served. The bottom line is that NI is moldable to an institution's scan techniques if proper steps are taken to find a desired noise threshold. Phantom scans should be acquired so that there is an understanding of how the ATCM will react under the institution's current CT settings.

Our initial scanning of the phantom, as well as work by others (9), showed that although NI may relate to image noise in water phantom scans, this relationship will not hold if parameters are changed. It also was not known how the NI relative to noise would react to the changes in density that would be seen as different parts of the patient were scanned with the same NI. The goal of the phantom scans was to determine our standard NI relative to image noise, as we tried to mold the NI to our CT parameters. This standard allowed us to ensure that the ATCM would be able to adjust appropriately to the density changes from one body part to another (e.g., thorax to abdomen). Although only 1 size of phantom was used in these scans, it is believed this factor was of little consequence because the goal of ATCM is to adjust to any size patient while maintaining the same noise level.

We had a unique starting point in the patient review data because there was a large database of patients who had been

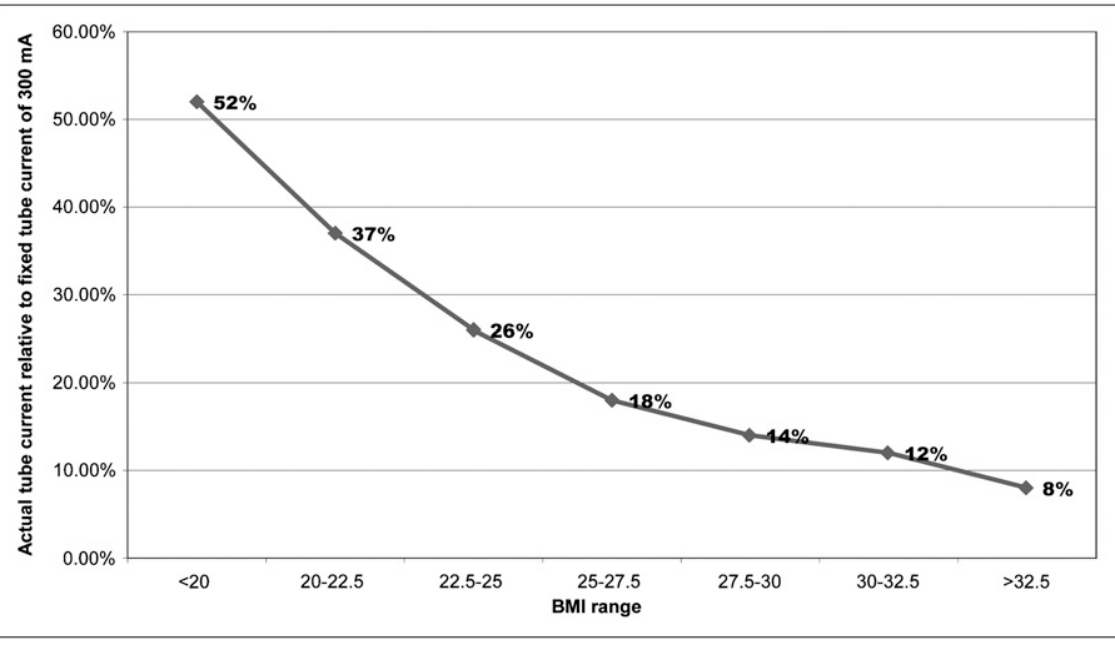

FIGURE 6. Average decrease in tube current by patient BMI group in CT scans using ATCM with $\mathrm{NI}$ of 20 compared with fixed tube current of $300 \mathrm{~mA}$. 
TABLE 1

Noise Levels in Thorax CT Images Using ATCM and NI of 20 in Patients with $\mathrm{BMI} \leq 28$

\begin{tabular}{|c|c|c|c|c|}
\hline \multirow[b]{2}{*}{ Patient no. } & \multicolumn{4}{|c|}{ Noise (SD of CT number in $\mathrm{HU}$ ) } \\
\hline & ROI 1 & ROI 2 & ROI 3 & Mean \\
\hline 1 & 11.7 & 9.3 & 12.9 & 11.3 \\
\hline 2 & 8.0 & 9.1 & 12.3 & 9.8 \\
\hline 3 & 8.5 & 14 & 10.5 & 11.0 \\
\hline 4 & 9.1 & 11.1 & 10.4 & 10.2 \\
\hline 5 & 7.1 & 10.2 & 11.9 & 9.7 \\
\hline 6 & 8.9 & 9.7 & 13.7 & 10.8 \\
\hline 7 & 9.1 & 8.4 & 12.6 & 10.0 \\
\hline 8 & 9.1 & 12 & 11.6 & 10.9 \\
\hline 9 & 10.0 & 12.4 & 13.1 & 11.8 \\
\hline 10 & 7.0 & 9.4 & 12.7 & 9.7 \\
\hline 11 & 9.4 & 11.3 & 13.2 & 11.3 \\
\hline 12 & 8.4 & 11 & 11.0 & 10.1 \\
\hline 13 & 7.0 & 12.2 & 14.4 & 11.2 \\
\hline 14 & 8.0 & 9.4 & 12.3 & 9.9 \\
\hline 15 & 7.3 & 10.8 & 12.2 & 10.1 \\
\hline 16 & 10.6 & 12.3 & 12.3 & 11.7 \\
\hline 17 & 10.8 & 10.6 & 11.0 & 10.8 \\
\hline 18 & 9.7 & 12.5 & 13.7 & 12.0 \\
\hline 19 & 9.3 & 11.8 & 14.0 & 11.7 \\
\hline 20 & 9.8 & 13.3 & 12.7 & 11.9 \\
\hline 21 & 7.5 & 10.3 & 12.4 & 10.1 \\
\hline 22 & 9.5 & 12.1 & 9.3 & 10.3 \\
\hline $\begin{array}{l}\text { Mean noise in all } \\
\text { thorax scans }\end{array}$ & & & & 10.7 \\
\hline
\end{tabular}

scanned with a fixed-tube-current protocol, allowing creation of a reference for noise, which was used in the ATCM protocol. In the postimplementation analysis, patients with higher BMIs generally had higher noise levels in their images than did patients with lower BMIs. Although BMI and noise corresponded moderately, differences in body habitus and tissue makeup made the relationship nonlinear. Because of ATCM's ability to account for the relationship between BMI and noise, our facility considers ATCM supe- rior to weight- or BMI-based fixed-tube-current protocols, as it can account for changes in body habitus that may not be intrinsic to BMI. This ability makes ATCM ideal for CT in PET/CT, because the CT covers multiple areas in a single sweep, possibly including coverage of the head, neck, and extremities.

One of our primary goals in ATCM initiation was to maintain image quality, measured by image noise, across the patient population. The set point for image noise was based on the physician's preferred level for noise tolerance. In our postimplementation patient analysis, it was deemed that the ATCM protocol maintained image quality across all our patient images in which the tube current modulated.

To maintain image quality, patients whose tube current does not modulate when scanned at a $300-\mathrm{mA}$ maximum must have their tube current increased, thus raising the radiation dose to these patients. This concern is addressed by implementing a maximum tube current of $400 \mathrm{~mA}$ for patients weighing over $120 \mathrm{~kg}$. Even with this action plan, peaking of the tube current modulation is observed in these patients, but the physicians and physicists think that the risks involved in countering this issue outweigh the benefits.

It is generally agreed in the radiographic community that the clearer the images, the greater their diagnostic value. Thus, most diagnostic imaging departments have a tendency to start out with a high-dose CT technique and are reluctant to decrease the settings. Several studies have shown, however, that marked radiation dose reductions result in minimal differences in diagnostic reliability (1316). For instance, Gollub et al. compared the diagnostic accuracy of a low-dose CT scan obtained during a PET/CT acquisition with that of a diagnostic CT scan obtained in their CT department (16). They found that most diagnostic misses resulted from the absence of intravenous or oral contrast material and that few were caused by image noise related to low tube current. Our facility has had similar experiences, although we may not see certain anatomic
FIGURE 7. Average image noise (SD of CT number in HU) in CT images of liver; $n=7$ for each BMI range. Noise levels were not markedly different for patients with BMls $<28$ whose examinations had tube current modulation.

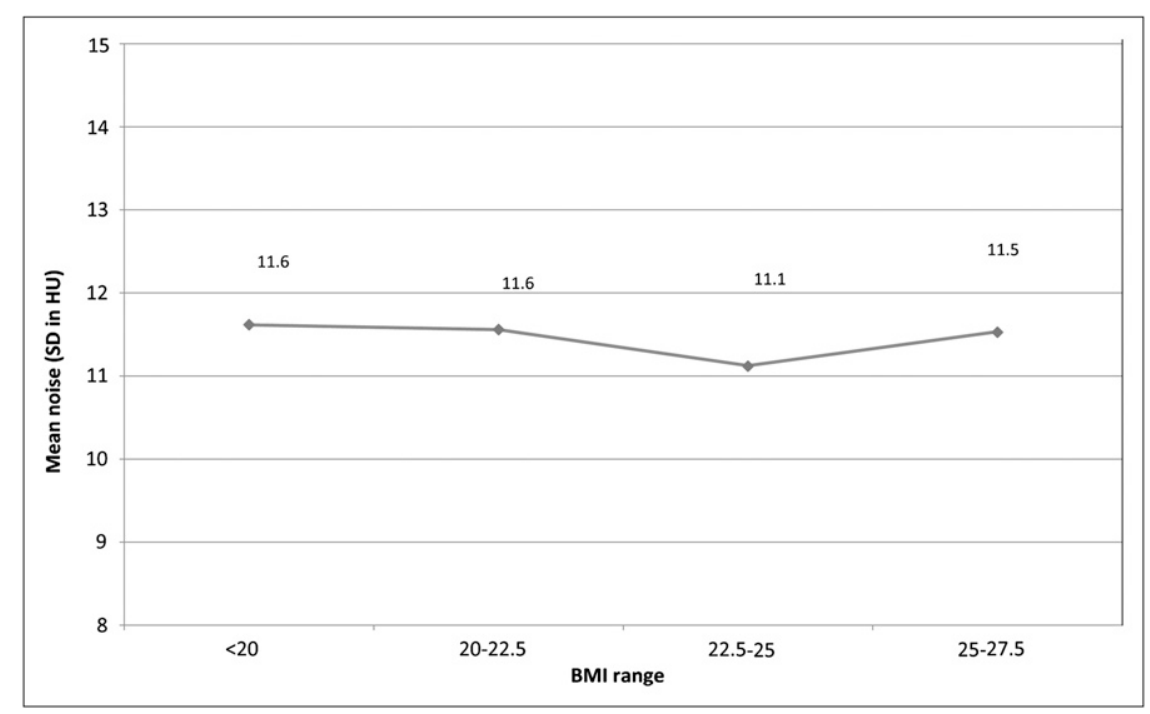


diseases because our examinations not only are done without contrast but also are free-breathing. It has been the physicians' experience that even at low tube currents they are able to see subcentimeter nodules and lymph nodes using the current ATCM protocol.

In addition to determining our ATCM parameters, the study tried to address a larger question in the radiology field: "How much image noise can be tolerated?" This question must be asked in each institution's radiology department, particularly as the number of diagnostic procedures increases. CT alone already accounts for approximately $50 \%$ of the total medical imaging radiation doses patients receive (17).

A recent study by Arch et al. showed promise of community awareness related to the efforts to reduce patients' radiation exposures (18). That 5-y follow-up study of members of the Society for Pediatric Radiology found a significant decrease in peak kilovoltage and tube current in pediatric protocols from the time of the initial survey. The authors attributed the dose reductions to increased consciousness of radiation risks. ATCM, along with iterative reconstructions, which are currently making their way into the marketplace, will help continue this trend of radiation dose reduction in all patient populations.

\section{Limitations}

The main limitation of this study was the use of subjective data (physician's image quality analysis) in a statistical analysis to determine a cutoff noise level. Although a consistent correlation was detailed between the physician's score and image noise, there was some variability. This variability would be even greater among multiple reading physicians based on this study, as some physicians may have less tolerance for noise than others. Therefore, a specific NI is not being proposed for adoption by other institutions. But rather a methodology is offered for other institutions to elect to follow to determine NIs for their ATCM protocol to limit radiation doses while maintaining image quality.

Another limitation of this study was the occurrence of motion artifacts in the descending aorta because of cardiac and respiratory motion. Motion artifacts may interfere with noise calculation, because 2 of the 3 thorax ROIs were in the $z$ plane. Thus, ROI noise measurements may be higher than if the ROIs were placed in the $x-y$ plane. In Table 1 for example, ROI 1 , which is placed in the $x-y$ plane, is consistently lower than the other 2 ROIs, placed in the $z$ plane. This variable was factored in by placing multiple ROIs and taking the average of the 3 for our final measurement. The study also yielded a solid agreement between the phantom study and the measurements found in the prospective patient analysis, leading us to believe that the detriment is limited. This study did not analyze the head and neck region. The standard practice is to perform separate scans of the torso and of the head and neck in patients who require both. There does seem to be some question about the CT scanner's ability to modulate the tube current in scans of the head and neck with the patient's arms up, because of the position of the arms in reference to the field of view.

\section{Considerations}

Bismuth shielding, especially for female patients' breasts, has proven to be an effective way to lower radiation doses by as much as $29 \%-40 \%$ without compromising image quality (19-21). However, care must be taken when implementing bismuth shielding along with ATCM. In the $x-, y$-, and $z$-axis modulation used by the Smart mA program, the shield is "seen" by the scanner during rotation, enabling the software to make on-the-fly adjustments and leading to a decrease in the radiation dose reduction provided by ATCM (18). In most GE Healthcare scanners, this dose increase may be avoided by using the Auto mA ( $z$ axis) function and placing the shield after the scout image is acquired. When the Auto mA program is used and the shield is placed after the scout image is acquired, the $z$-axis modulation is prescribed on the scout image, preventing tube current adjustments during the scan (10). The modification allows the system to prescribe the radiation dose without taking the shield into consideration.

\section{CONCLUSION}

ATCM is an effective tool for tailoring the CT radiation dose to a patient's body habitus. Phantom measurements are valid for predicting tube current modulation and noise in patient studies. Thus, prospectively selecting an NI is possible if a physician's preferred noise level is known and the same technique is used in both clinical and phantom applications.

\section{REFERENCES}

1. Di Carli MF, Dorbala S, Meserve J, El Fakhri G, Sitek A, Moore SC. Clinical myocardial perfusion PET/CT. J Nucl Med. 2007;48:783-793.

2. Fletcher JW, Djulbegovic B, Soares HP, et al. Recommendations on the use of ${ }^{18}$ F-FDG PET in oncology. J Nucl Med. 2008;49:480-508.

3. Czernin J, Benz MR, Allen-Auerbach MS. PET/CT imaging: the incremental value of assessing the glucose metabolic phenotype and the structure of cancers in a single examination. Eur J Radiol. 2010;73:470-480.

4. Schneider J. Growth rate for PET procedure volume slows to single digits. Aunt Minnie Web site. Available at: http://www.auntminnie.com/index.asp?sec=ser\&sub= def\&pag $=$ dis\&ItemID =84616. Accessed January 25, 2011.

5. Brix G, Lechel U, Glatting G, et al. Radiation exposure of patients undergoing whole-body dual-modality ${ }^{18}$ F-FDG PET/CT examinations. J Nucl Med. 2005;46: 608-613.

6. Frush DP, Soden B, Frush KS, Lowry C. Improved pediatric multidetector body CT using a size-based color-coded format. AJR. 2002;178:721-726.

7. Livingstone RS, Dinakaran PM, Cherian RS, Eapen A. Comparison of radiation doses using weight-based protocol and dose modulation techniques for patients undergoing biphasic abdominal computed tomography examinations. J Med Phys. 2009;34:217-222.

8. Hur G, Hong SW, Kim SY, et al. Uniform image quality achieved by tube current modulation using SD of attenuation in coronary CT angiography. AJR. 2007; 189:188-196.

9. Rampado O, Izzo E, Garelli CC, Bianchi G, Gandini R Ropolo. Effective dose and image quality evaluations of an automatic CT tube current modulation system with an anthropomorphic phantom. Eur J Radiol. 2009;72:181-187.

10. Kalra MK, Maher MM, Toth TL, et al. Techniques and applications of automatic tube current modulation for CT. Radiology. 2004;233:649-657. 
11. Kalra MK, Rizzo S, Maher MM, et al. Chest CT performed with z-axis modulation: scanning protocol and radiation dose. Radiology. 2005;237:303-308.

12. Funama Y, Taguchi T, Awai K, Sakabe D, Shimamura M, Kamshita Y. Image noise and radiation dose using an automatic tube current modulation technique at 64-detector computed tomography: effect of off center patient position, bowtie filter type, and scan projection radiograph. J Comput Assist Tomogr. 2009;33: 973-977.

13. Ciaschini MW, Remer EM, Baker ME, Lieber M, Herts BR. Urinary calculi: radiation dose reduction of $50 \%$ and $75 \%$ at CT-effect on sensitivity. Radiology. 2009;251:105-111.

14. Fisichella VA, Bath M, Allansdotter JA, et al. Evaluation of image quality and lesion perception by human readers on 3D CT colonography: comparison of standard and low radiation dose. Eur Radiol. 2009;20:630-639.

15. Mulkens TH, Marchal P, Daineffe S, et al. Comparison of low-dose with standard-dose multidetector CT in cervical spine trauma. AJNR. 2007;28:1444-1450.
16. Gollub MJ, Hong R, Sarasohn DM, Akhurst T. Limitations of CT during PET/CT. J Nucl Med. 2007;48:1583-1591.

17. Einstein AJ. Medical imaging: the radiation issue. Nat Rev Cardiol. 2009;6:436-438.

18. Arch ME, Frush DP. Pediatric body MDCT: a 5-year follow-up survey of scanning parameters used by pediatric radiologists. AJR. 2008;191:611-617.

19. Coursey C, Frush D, Yoshizumi T, Toncheva G, Nguyen G, Greenberg SB. Pediatric chest MDCT using tube current modulation: effect on radiation dose with breast shielding. AJR. 2008;190:W54-W61.

20. Fricke BL, Donnelly LF, Frush DP, et al. In-plane bismuth breast shields for pediatric CT: effects on radiation dose and image quality using experimental and clinical data. AJR. 2003;180:407-411.

21. Yilmaz MH, Albayram S, Yaşar D, et al. Female breast radiation exposure during thorax multidetector computed tomography and the effectiveness of bismuth breast shield to reduce breast radiation dose. J Comput Assist Tomogr. 2007;31:138-142. 